\title{
LOW AMPLITUDE NEW TYPE VARIABLE STARS IN
}

\section{GLOBULAR CLUSTERS}

\author{
YAO BAO-AN \\ Shanghai Observatory \\ ZHANG CHL NG-SHENG and QIN DAO \\ Purple Mountain Observatory \\ and \\ TONG JIAN-HUA \\ Beijing Observatory
}

Rapid progress in the stellar pulsation research has presented many new challenges to traditional pulsation theory. The following progress made by us should belong to one of the new challenges:

1) There are many variable stars located at the Horizontal Branch (HB) but outside the instability strip with amplitude larger than 0.02 mag.

Maybe some astronomers have already doubted of the 1950's conclusionthe RR Lyrae stars are confined to the narrow instability strip in the C$M$ diagram, the boundaries of the gap are extremely sharp and definite, beyond the edges no light variations occur with ranges greater than 0.02 mag. We are changing the conclusion by observations. Here we do not mean the microvariability. We still raise the question from the classical viewpoint. We want to show that there are variable stars outside the strip with peak to peak amplitudes larger than $0.02 \mathrm{mag}$.

Since 1975 we use M4 as the calibration cluster to measure our newly discovered flare stars and variable stars in the Oph dark cloud region. Because we use the double astrograph and the Schmidt telescope together, three plates were got simultaneously. As the by-product a large number of unusual suspected variable stars were found (1979; Yao et al. 1981). There are 12 stars located at the $\mathrm{HB}$ but blueward of the instability strip and 7 stars at HB but redward of the gap. All of them are the members of M4 (Cudworth 1990). We have checked 3 of them with the RCA CCD attached to the Zeiss 1-meter telescope of Yunnan Observatory and published the preliminary results (Yao 1987). (G206, G140, G481). Another star G327, was confirmed recently with the 60-cm reflector of MSSSO (Yao 1991). We believe that most of the other stars in our list are variable too because we chose the above 4 stars randomly and all of them appeared to be variables. Some of them have been observed with the CCD camera and the reduction is progressing. In fact other astronomers also pointed out the light variation of some stars common in our list, e.g. G537, 539, 258, 246 and 172 (Evans 1977; Alcaino 1975). According to our photographic observation none of them be- 
longs to the normal RR Lyrae stars. We hope that we will determine their periods.

2) There are variable stars at the tip of the RGB but leftward of the region occupied by long period variables.

R. E. White et al. (1975) gave evidence for variability of some stars located at the tip of RGB but leftward of the region occupied by Mira or long period variable stars (further down the giant and asymptotic-giant branches) in M15 and other globular clusters. But in an attempt to confirm and extend White's results D.E. Welty (1985) obtained photographic photometry of giant and asymptotic-giant branch stars in six globular clusters (M3, M5, M13, M15, M71, M92) by measuring several hundred plates of these clusters on the PDS. He did not confirm variability for any of these stars found by White et al. with amplitude $>0.2 \mathrm{mg}$. Obviously, further investigation of these stars is significant for understanding the stellar evolution on the giant and asymptotic-giant branches. However, we have found that the red giant star K1040 in M15 located at the same region has a period of $\sim 4.3$ hour with amplitude $\sim 0.04 \mathrm{mag}$ (Yao, 1990). The similar star G512 in M4 was published by us before (Yao et al. 1981). We share White's opinion and believe this is the common phenomena among these kind of stars. We are searching for the similar stars in globular clusters too.

3) There are variables located at the lower middle part of the RGB.

We have shown that there are variable stars located at the lower middle part of the red giant branch in the globular cluster M4(1979). G265 and G543 have shown certain periodicity (Yao, 1987). There are 7 similar stars in our list in M4 to be confirmed by CCD photometry. No results were reported in the past so the theorist did not pay attention to it. We will continue to observe them in the future.

4) Are the constant stars located within the gap of $\mathrm{HB}$ really constant?

People have already found a few stars located at the middle part of the instability strip of the horizontal branch in several globular clusters which do not show evidence of light variation. G392 in M4 is such a star (Cudworth 1990). We suggest that people should reobserve these stars with high accuracy because we have already found in 1979 that the G392 is really a variable star. Due to the low precision of the photographic photometry we have not got its period. It is not a normal $R R$ Lyrae star (with amplitude $<0.2$ mag and maybe multiperiodic). We are observing this star with CCD camera and try to get its periods soon.

5) The UV bright star K1082 in M15 is a short period variables indeed.

Using the photographic plates obtained with the $60-\mathrm{cm}$ Zeiss reflector of P.M.O. by Kukarkin et al., Chu (1977) has reported that the UV Bright star K1082 in M15 is a new type variables with a period $P=0.087004$ day and amplitude of $0.2 \mathrm{mag}$. However, the later observations (Hesser et al., 1979; Liller et al., 1980; Smith et al., 1979) show no evidence of variation 
with the reported period and amplitude. Due to the potential importance of the star, we have scanned the original plates again with the PDS of P.M.O. Our results have shown that it can be said with $90 \%$ probability that $\mathrm{K} 1082$ is a new type variable star. But the reported period is wrong. It may be a multi-period variables with the main period $P_{1}=0.1683$ day. It is possible that the second period $P_{2}=0.0985$ or 0.0437 day.

We have also observed the star with CCD photometer. The amplitude of the light variation is less than $0.01 \mathrm{mag}$ in 1987 and $\sim 0.04$ mag in 1991 and the period is changing. The reduction is still in progress and we will continue to monitor $\mathrm{K} 1082$. 